\title{
Flux tube with dynamical fermions from high temperature SU(3) lattice gauge theory
}

\section{Sodbileg Chagdaa*t}

Department of Theoretical Physics, Institute of Physics and Technology, Mongolian Academy of Sciences, Peace Ave. 54b, 13330 Ulaanbaatar, Mongolia

E-mail: sodbilegch@mas.ac.mn

\section{Enkhtuya Galsandorj}

Department of Theoretical Physics, Institute of Physics and Technology, Mongolian Academy of Sciences, Peace Ave. 54b, 13330 Ulaanbaatar, Mongolia

E-mail: enkhtuyag@mas.ac.mn

\section{Battogtokh Purev}

Department of Theoretical Physics, Institute of Physics and Technology, Mongolian Academy of Sciences, Peace Ave. 54b, 13330 Ulaanbaatar, Mongolia

E-mail: battogtokhp@mas.ac.mn

\section{Olaf Kaczmarek}

Fakultät für Physik, Universität Bielefeld, D-33615 Bielefeld, Germany

Institute of Particle Physics, Central China Normal University, 152 LuoYu Road, Wuhan 430079

E-mail: okacz@physik.uni-bielefeld.de

\section{Heng-Tong Ding}

Institute of Particle Physics, Central China Normal University, 152 LuoYu Road, Wuhan 430079

E-mail: hengtong.ding@mail.ccnu.edu.cn

We explore the profiles of the flux tube connecting a quark and an antiquark in high temperature SU(3) lattice gauge theory in close vicinity to the critical temperature of the phase transition. In this work, we consider the more realistic case of the flux tube with dynamical quarks, extending the previous study to SU(3) gauge group and making use of the Gradient flow method in smoothing procedure for noise reduction. The profiles of the chromoelectric and chromomagnetic field strengths in the flux tube have been measured from Polyakov loop-plaquette correlations using the highly improved staggered quark (HISQ) action on a lattice with temporal extent $N_{\tau}=8$. We present preliminary results for distances up to $2.5 \mathrm{fm}$ and temperatures up to $1.09 T_{c}$.

37th International Symposium on Lattice Field Theory - Lattice2019

16-22 June 2019

Wuhan, China

\footnotetext{
* Speaker.

$\dagger$ This research is carried out under the project with contract number "SHuSs 2017/28", with the approval and support of the Ministry of Education, Culture, Science and Sports of Mongolia and Mongolian Foundation for Science and Technology. This work was partly supported by the DFG (German Research Foundation) - project number 315477589 - TRR 211. We are grateful to the HotQCD collaboration for providing us with the dynamical fermion configurations. We also thank Bielefeld colleagues for the so called ParallelGPUCode program of the Gradient flow method. We acknowledge CCNU colleagues for their financial support and fruitful discussion on physics. Flux tube measurements and analysis have been performed at the Institute of Physics and Technology of Mongolian Academy of Sciences.
} 


\section{Introduction}

The strong interaction of matter, one of the four fundamental forces of nature, provides the basic force which is responsible for binding elementary particles together to form nuclear matter. In particular, quarks and gluons appear to be confined in ordinary matter due to the mechanism of color confinement, which is one of the most challenging and not yet fully explained physics phenomena of the strong interaction. Reaching a detailed understanding of color confinement mechanism is one of the central goals of experimental as well as theoretical high energy physics as they are relevant for heavy ion collision experiment and for cosmology.

Mediators of the strong interaction are gluons and due to the non-abelian nature of the gauge interactions in QCD they form a narrow tube of chromoelectric flux between two quarks. There are numerous accurate lattice simulations that have made considerable progress in simulation of the flux tubes, particularly, in the cases of SU(2) and SU(3) pure gauge theories at zero and finite temperatures [1-9] all concerning to address the flux tube behavior at zero temperature as well as close vicinity to the critical temperature of the deconfinement phase transition. But there has been little progress in understanding the flux tube behavior in the presence of dynamical fermions due to the non-locality of the effective lattice action for the gluonic gauge fields. In the effective gauge action the logarithm of the determinant of the fermion matrix appears which becomes more and more non-local for fermions with small mass. In order to avoid this problem early simulations used quenched approximation, but they can not represent the real world.

In this work we study the profiles of the distribution of chromoelectric and chromomagnetic field components, energy density and width of the flux tube around the deconfinement phase transition in QCD with (2+1) flavors, using HISQ/tree action on a lattice exploiting the Gradient flow method for noise reduction. We work on the line of constant physics, where the strange quark mass is fixed to its physical value $m_{s}$ at each value of the gauge coupling and the strange to light quark mass ratio is $m_{s} / m_{l}=27$. We present some new preliminary results for interquark distances up to $2.5 \mathrm{fm}$ and temperatures up to $1.09 T_{c}$.

\section{Gradient flowed configurations}

Field configurations with dynamical quarks are taken from HotQCD collaboration. With the highly improved staggered quark action with tree level improved Symanzik gauge action (HISQ/tree) 6000 - 9000 gauge field configurations were generated for each value of the five gauge couplings on the lattice of size $32^{3} \times 8$ using RHMC algorithm based on the simple molecular dynamics. In order to reduce the autocorrelation time, the configurations were taken after 10 sweeps. Further we collected from the data one configuration from each five configurations reducing the total number of it to about 1150 so that a single GPU in the institute of Ulaanbaatar can make the generated configurations flowed by means of the Gradient flow method encoded in the so called ParallelGPUCode program [10].

The generated configurations have been flowed by the flow equation $[11,12]$

$$
\dot{U}_{t}(x, \mu)=-g_{0}^{2}\left\{\partial_{x, \mu} S_{W}\left(U_{t}\right)\right\} U_{t}(x, \mu),\left.\quad U_{t}(x, \mu)\right|_{t=0}=U(x, \mu),
$$


which turned out to be a very efficient computational strategy to increase signal-to-noise ratio in lattice numerical simulation. It introduces extra coordinate $t$ called flow-time and the gauge fields along the flow become smoother with the smearing radius $r_{\text {smear }}=\sqrt{8 t}$.

If $\partial_{x, \mu}$ is a SU(3)-valued differential operator, calculation of $\partial_{x, \mu} S_{W}\left(U_{t}\right)$ gives [10]

$$
g_{0}^{2} \partial_{x, \rho} S_{W}\left(U_{t}\right)=\frac{1}{2}\left(\Omega(x, \rho)-\Omega^{\dagger}(x, \rho)\right)-\frac{1}{6} \operatorname{tr}\left(\Omega(x, \rho)-\Omega^{\dagger}(x, \rho)\right)
$$

where $\Omega(x, \rho)=U(x, \rho) W^{\dagger}(x, \rho)$ with the staple $W_{\rho}^{\dagger}(x)$. Expression in the right hand side of the Eq. (2.2) have been constructed from the link variables on the lattice and it makes it convenient to solve the Eq. (2.1) numerically for link smearing.

The gauge field configurations are flowed in the range $0.01-0.25$ of the flow-time value with the step of 0.01 . And we have done the measurements at the eight values of them. When the flowtime increases all the four components are reaching their plateau values at some point of $t$. That point seems to be $t=0.15$ at all temperatures in our case. Indeed our data at the first five values of the flow-time gave the bad signal-to-noise ratio, while those at the last three which are $t=0.15$, 0.20 and 0.25 gave the good one. As this holds for all temperatures and distances, we choose to show the results from $t=0.25$ only in this paper.

\section{Flux tube measurement}

In our flux tube simulation program Polyakov loop-plaquette correlation operators [13,14]

$$
f_{\mu v}(R, \mathbf{x})=\frac{\beta}{a^{4}}\left[\frac{\left\langle L(0) L^{+}(R) \square_{\mu v}(\mathbf{x})\right\rangle}{\left\langle L(0) L^{+}(R)\right\rangle}-\left\langle\square_{\mu v}\left(\mathbf{x}_{\mathrm{ref}}\right)\right\rangle\right]
$$

measure the chromofield components from the Gradient flowed configurations. $f_{\mu \nu}$ is chromofield strength where $f_{12}, f_{13}$ and $f_{23}$ are the space-space components and define chromomagnetic contributions to the chromofield strength, while $f_{24}, f_{34}$ and $f_{14}$ are space-time components that define chromoelectric contributions to the chromofield strength. $\beta$ and $a$ is the coupling constant and lattice spacing respectively. The time propagation of a quark and an antiquark at distance $R$ from each other is represented by the Polyakov loop $L(0)$ and its conjugate $L^{+}(R)$, where a Polyakov loop at some location $\vec{n}$ is

$$
L(\vec{n}) \equiv \frac{1}{N_{c}} \operatorname{Tr} \prod_{\tau=1}^{N_{\tau}} U_{4}(\vec{n}, \tau) .
$$

The plaquette variable $\square_{\mu v}=\frac{1}{N_{c}} \operatorname{Tr}\left(U_{\mu, v}\right)$ at distance $\mathbf{x}$ from the axis connecting the two sources correlates with the two Polyakov loops and thus varying the position of the plaquette, $\mathbf{x}\left(\mathbf{x}_{\|}, \mathbf{x}_{\perp}\right)$, we are able to scan distribution surface of the chromofield strength $f_{\mu \nu}$ around quark-antiquark pair. One can then investigate various profiles of the distribution surface by changing profile coordinate with respect to the axis parallel as well as perpendicular to the plane containing the quark-antiquark pair.

Simulations have been performed at five values of the gauge coupling $\beta$ in the range $6.390-$ 6.500 which corresponds to physical temperatures $T$ in the range $0.97 T_{c}-1.09 T_{c}$. We measured even numbers of lattice distance $R / a$ in the range $4-16$ which give the physical distances ranging from $0.5 \mathrm{fm}$ to $2.5 \mathrm{fm}$. The Jackknife method has been used to get the estimates of the statistical errors. 


\section{Scaling function}

To set the lattice spacing we used $f_{K}$ scaling as determined in Appendix B of [15] with the coefficients $c_{0}^{K}=7.49415, c_{2}^{K}=46049$ and $d_{2}^{K}=3671$ as updated by 2018 . Here one fits $a f_{K}$ data to the Ansatz

$$
a f_{K}(\beta)=\frac{c_{0}^{K} f(\beta)+c_{2}^{K}(10 / \beta) f^{3}(\beta)}{1+d_{2}^{K}(10 / \beta) f^{2}(\beta)},
$$

where

$$
f(\beta)=\left(b_{0}(10 / \beta)\right)^{-b_{1} /\left(2 b_{0}^{2}\right)} \exp \left(-\beta /\left(20 b_{0}\right)\right)
$$

and $b_{0}$ and $b_{1}$ are the coefficients of the universal two-loop beta functions. Then we can express lattice spacing in $\mathrm{fm}$

$$
a(\beta)=a f_{K}(\beta) \cdot \frac{197.3 \mathrm{MeV} \cdot \mathrm{fm}}{156.1 / \sqrt{2} \mathrm{MeV}}
$$

and temperature in $\mathrm{MeV}$

$$
T\left(\beta, N_{\tau}\right)=\frac{156.1 / \sqrt{2} \mathrm{MeV}}{a f_{K}(\beta) \cdot N_{\tau}} .
$$

Now one can obtain estimates of the corresponding temperatures in units of the critical temperature at each gauge coupling by the ratio

$$
\frac{T}{T_{c}}=\frac{T\left(\beta, N_{\tau}\right)}{T_{c}\left(\beta_{c}, N_{\tau}\right)} \equiv \frac{a f_{K}\left(\beta_{c}\right)}{a f_{K}(\beta)} .
$$

\section{Results}

\subsection{Energy density in the flux tube}

The six chromofield strength components compose energy density that gives the physical flux tube. In order to investigate the behavior of the real physical flux tube across the deconfinement phase transition it is worth to consider various profiles of the energy density and their parameter dependence. Total magnetic and electric chromofield strengths which are, respectively,

$$
\mathscr{M}=-\left(f_{12}+f_{13}+f_{23}\right) \quad \text { and } \quad \mathscr{E}=f_{24}+f_{34}+f_{14}
$$

define the total energy density as

$$
\varepsilon=\mathscr{E}+\mathscr{M} .
$$

We were able to obtain longitudinal and transverse profiles of the energy density $\varepsilon$ calculated with the Eq. (5.2) at several values of interquark distance and temperature. The Fig. 1 presents energy density value at the point $\left(x_{\|}=R / 2, x_{\perp}=0\right)$ of those profiles as functions of the interquark distance and temperature. The midpoint belongs to the central region of our interest between the two sources. The first plot shows that the energy density in the middle region between the two sources clearly decreases with increasing distance and vanishes at about $R=2 \mathrm{fm}$. The second plot shows its temperature dependence, from that one can see that in the confinement phase the energy density of the flux tube decreases when temperature approaches $T_{c}$ from below. In the deconfinement phase the energy density further decreases with temperature at all distances. We see from the plot that 

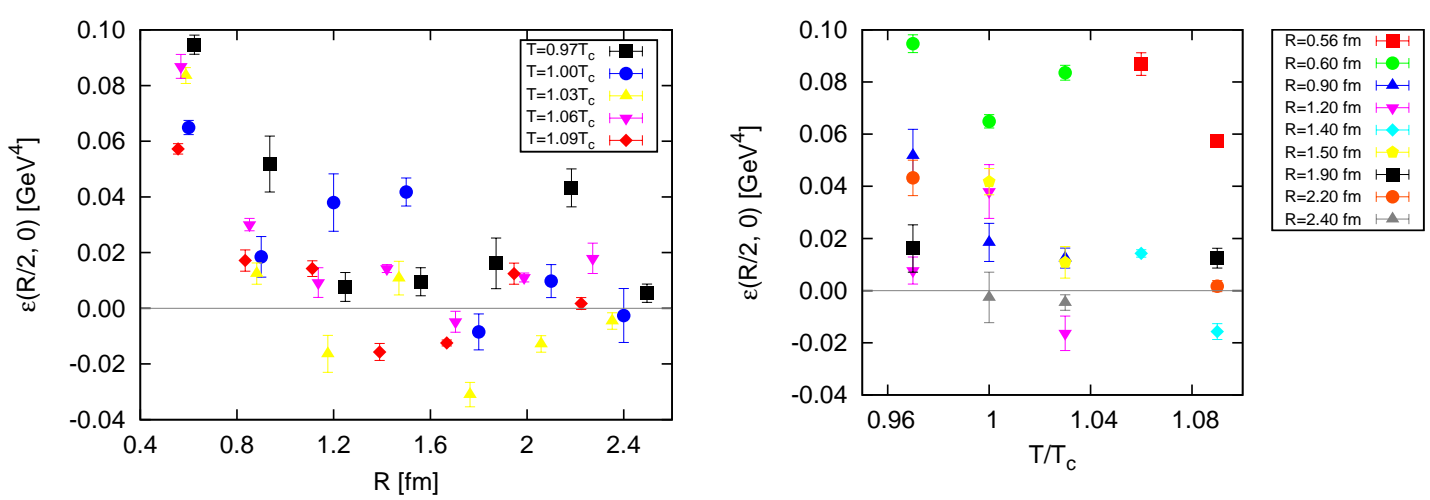

Figure 1: The energy density value at the midpoint $\left(x_{\|}=R / 2, x_{\perp}=0\right)$ between a quark and an antiquark as functions of the interquark distance and temperature from the lattice of size $32^{3} \times 8$.

the flux tubes with length of less than $2.2 \mathrm{fm}$ do still survive for temperatures up to $1.09 T_{c}$. Thus we find string breaking distance to be around $R=2.2 \mathrm{fm}$ in the region with temperature $1.09 T_{c}$ in full QCD. This might be suggesting that flux tube in full QCD at high temperature but just above $T_{c}$ can be similar to its zero temperature case studied in [8] where no string breaking observed up to the distance $R \approx 2 \mathrm{fm}$.

\subsection{Width of the flux tube}

We define physical width of the flux tube as width of its energy density and estimate it quantitatively by fitting the middle transverse distribution of the energy density to an exponential function [16]

$$
f\left(x_{\perp}\right)=a_{1} e^{-a_{2} x_{\perp}}+K
$$

from that the width $D_{\varepsilon}$ has been computed via

$$
D_{\varepsilon}^{2} / a^{2}=\frac{\int d^{2} x_{\perp} x_{\perp}^{2}\left(f\left(x_{\perp}\right)-K\right)}{\int d^{2} x_{\perp}\left(f\left(x_{\perp}\right)-K\right)}
$$

in terms of the fitting parameters $a_{1}$ and $a_{2} . K$ is an unphysical parameter which is due to the statistical fluctuations of the fields at the reference point $\mathbf{x}_{\text {ref }}$ of the Eq. (3.1) and due to its finite distance from the center of the flux tube [17]. The fit is done for each five gauge coupling $\beta$ and seven lattice distances $R / a=4,6,8,10,12,14,16$ for each gauge coupling. We choose those $R=N a$ distances with $N$ to be of an even number in order to fit the transverse profiles on the middle point $x_{\|}=R / 2$ between the two sources.

The resulting width of the energy density, $D_{\varepsilon}$, is displayed in the Fig. 2 in physical units as functions of the distance $R$ and temperature $T$. Width of the flux tube increases with interquark distance until the distance reaches around $0.9 \mathrm{fm}$, after which it strongly decreases with further increasing distance. The presence of dynamical quarks seems to widen the flux in a small distance up to around $R=0.9 \mathrm{fm}$ but to suppress it with further separation which could be an indication of hadronization. This looks in contrast with the case of SU(3) pure gauge theory [17] where one finds a monotone or a weak logarithmic spreading of the flux tube as a function of interquark distance. 

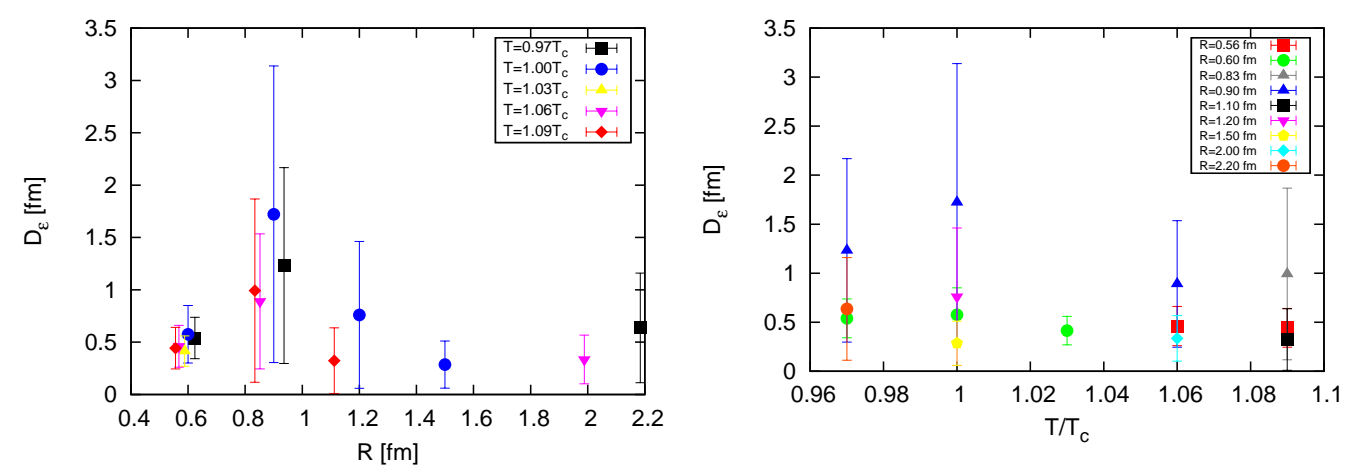

Figure 2: The width of the flux tube as functions of the interquark distance and temperature from the lattice of size $32^{3} \times 8$.

The strong decrease with distance after $R=0.9 \mathrm{fm}$ could indicate strong localization of the QCD string yet we observed no string breaking up to around $R=2.2 \mathrm{fm}$ in the deconfinement phase.

Also our results confirm the earlier result of very first study of the breaking of the flux tube in full QCD by [18] in that both our and their study showing hadronization beyond certain distance. Difference is that our results show that this hadronization takes place in the both two phases, confinement and deconfinement, at least up to temperature $1.09 T_{c}$. We see that the temperature accelerates the rate of the hadronization probably causing an additional polarization effects due to the dynamical fermions. Temperature dependence of the width at a fixed distance is shown in the second plot of the Fig. 2 for various distances. We see that the width of the flux tube increases as a function of temperature up to $T_{c}$. In the deconfinement phase it tends to decrease with temperature yet shows non-vanishing values at our largest temperature.

\section{Conclusion}

We find that the width of the flux tube does not always increase as a function of distance in both confined and deconfined phases when the dynamical fermions are presented. In both phases it increases with distance up to around $R=0.9 \mathrm{fm}$ after which it is strongly suppressed due to the dynamical fermions. This result supports [18]'s result that is in the confined phase and [17]'s result that is in the deconfined phase. Our present study has advantages as it is updating the pioneer work [18] with the modern computational strategies and differing from [17] in accounting for dynamical fermions.

The decreases of the both energy density and width of the flux tube with temperature above the $T_{c}$ suggest that flux tube structure melts eventually beyond certain distance and temperature value in the deconfined phase. Our study in the presence of dynamical fermions shows that flux tube structures with length of $2 \mathrm{fm}$ persists up to temperature $1.06 T_{c}$ and flux tubes with length of $1.1 \mathrm{fm}$ persists up to temperature $1.09 T_{c}$. These are in qualitative agreement with the conclusion in [7] that the flux tube structure survives to the deconfinement transition and conclusion in [19] that in presence of dynamical fermions for a sufficiently large distance between sources the flux 
tube structure disappears. In future study we plan to increase the statistics, extend the temperature range, improve the fit function and compute relevant observables of interest.

\section{References}

[1] G. S. Bali, K. Schilling and Ch. Schlichter, Observing long color flux tubes in SU(2) lattice gauge theory, Phys. Rev. D51 (1995) 5165-5198, [hep-lat/ 9409005 ].

[2] R. W. Haymaker, V. Singh, Y. Peng and J. Wosiek, Distribution of the color fields around static quarks: Flux tube profiles, Phys.Rev. D53 (1996) 389-403, [hep-lat/9406021].

[3] M. Lüscher, G. Münster and P. Weisz, How thick are chromo-electric flux tubes?, Nucl. Phys. B180 [FS2] (1980) 1-12.

[4] A. Allais and M. Caselle, On the linear increase of the flux tube thickness near the deconfinement transition, JHEP 01 (2009) 073, [hep-lat/0812.0284].

[5] M. Caselle, Flux tube delocalization at the deconfinement point, JHEP 08 (2010) 063 , [hep-lat/1004.3875].

[6] M. Caselle and P. Grinza, On the intrinsic width of the chromoelectric flux tube in finite temperature LGTs, JHEP 11 (2012) 174, [hep-lat/1207.6523].

[7] P. Cea, L. Cosmai, F. Cuteri and A. Papa, Flux tubes at finite temperature, JHEP 06 (2016) 033, [hep-lat/1511.01783].

[8] H. Ichie, V. Bornyakov, T. Streuer and G. Schierholz, Flux tubes of two- and three-quark system in full QCD, Nucl. Phys. A721 (2003) 899-902, [hep-lat/0212036].

[9] P. Bicudo, N. Cardoso and M. Cardoso, Pure gauge QCD flux tubes and their widths at finite temperature, [hep-lat/1702.03454].

[10] L. Mazur, Applications of the Gradient flow method in lattice QCD, Master's thesis, Bielefeld University (2017).

[11] M. Lüscher, Properties and uses of the Wilson flow in lattice QCD, JHEP 08 (2010) 071, [hep-lat/1006.4518].

[12] M. Lüscher, Trivializing maps, the Wilson flow and the HMC algorithm, Commun. Math. Phys. 293 (2010) 899-919, [hep-lat/0907.5491].

[13] M. Fukugita and T. Niuya, The distribution of chromoelectric flux in SU(2) lattice gauge theory, Phys. Lett. 132B (1983) 374-378.

[14] S. Chagdaa, E. Galsandorj, E. Laermann and B. Purev, Width and string tension of the flux tube in SU(2) lattice gauge theory at high temperature, J. Phys. G 45 (2017) 025002.

[15] A. Bazavov et al., The QCD transition temperature: results with physical masses in the continuum limit II, JHEP 06 (2009) 088, [hep-lat/ 0903 . 4155].

[16] R. Sommer, Chromoflux distribution in lattice QCD, Nucl. Phys. B291 (1987) 673-691.

[17] P. Bicudo, N. Cardoso and M. Cardoso, Pure gauge flux tubes and their widths at finite temperature, Nucl. Phys. B940 (2019) 88-112.

[18] W. Feilmair and H. Markum, Flux tube profiles with dynamical quarks and finite temperatures, Nucl. Phys. B370 (1992) 299-309.

[19] L. Cosmai, P. Cea, F. Cuteri and A. Papa, Flux tubes in QCD with (2+1) HISQ fermions, PoS (LATTICE2016) 344 (2016), [hep-lat/1701.03371]. 